\section{IMAGE Resolution Enhancement by Using Discrete and Stationary Wavelet Decomposition}

Hasan Demirel and Gholamreza Anbarjafari

\begin{abstract}
In this correspondence, the authors propose an image resolution enhancement technique based on interpolation of the high frequency subband images obtained by discrete wavelet transform (DWT) and the input image. The edges are enhanced by introducing an intermediate stage by using stationary wavelet transform (SWT). DWT is applied in order to decompose an input image into different subbands. Then the high frequency subbands as well as the input image are interpolated. The estimated high frequency subbands are being modified by using high frequency subband obtained through SWT. Then all these subbands are combined to generate a new high resolution image by using inverse DWT (IDWT). The quantitative and visual results are showing the superiority of the proposed technique over the conventional and state-of-art image resolution enhancement techniques.
\end{abstract}

Index Terms-Discrete wavelet transform, image super resolution, stationary wavelet transform.

\section{INTRODUCTION}

Resolution has been frequently referred as an important aspect of an image. Images are being processed in order to obtain more enhanced resolution. One of the commonly used techniques for image resolution enhancement is Interpolation. Interpolation has been widely used in many image processing applications such as facial reconstruction [1], multiple description coding [2], and super resolution [3]-[6]. There are three well known interpolation techniques, namely nearest neighbor interpolation, bilinear interpolation, and bicubic interpolation.

Image resolution enhancement in the wavelet domain is a relatively new research topic and recently many new algorithms have been proposed [4]-[7]. Discrete wavelet transform (DWT) [8] is one of the recent wavelet transforms used in image processing. DWT decomposes an image into different subband images, namely low-low (LL), lowhigh (LH), high-low (HL), and high-high (HH). Another recent wavelet transform which has been used in several image processing applications is stationary wavelet transform (SWT) [9]. In short, SWT is similar to DWT but it does not use down-sampling, hence the subbands will have the same size as the input image.

In this work, we are proposing an image resolution enhancement technique which generates sharper high resolution image. The proposed technique uses DWT to decompose a low resolution image into different subbands. Then the three high frequency subband images have been interpolated using bicubic interpolation. The high frequency subbands obtained by SWT of the input image are being incremented into the interpolated high frequency subbands in order to correct the estimated coefficients. In parallel, the input image is also interpolated separately. Finally, corrected interpolated high frequency subbands and interpolated input image are combined by using inverse DWT (IDWT) to achieve a high resolution output image. The proposed technique has been compared with conventional and state-of-art image resolu-

Manuscript received May 28, 2010; revised August 09, 2010; accepted October 05, 2010. Date of publication October 18, 2010; date of current version April 15, 2011. The associate editor coordinating the review of this manuscript and approving it for publication was Dr. Rafael Molina.

H. Demirel is with the Department of Electrical and Electronic Engineering, Eastern Mediterranean University, Mersin 10, Turkey (e-mail: hasan.demirel@emu.edu.tr).

G. Anbarjafari is with the Department of Information Systems Engineering, Cyprus International University, Mersin 10, Turkey (e-mail: sjafari@ ciu.edu.tr).

Digital Object Identifier 10.1109/TIP.2010.2087767 tion enhancement techniques. The conventional techniques used are the following:

— interpolation techniques: bilinear interpolation and bicubic interpolation;

— wavelet zero padding (WZP).

The state-of-art techniques used for comparison purposes are the following:

— regularity-preserving image interpolation [7];

— new edge-directed interpolation (NEDI) [10];

— hidden Markov model (HMM) [11];

- HMM-based image super resolution (HMM SR) [12];

- WZP and cycle-spinning (WZP-CS) [13];

- WZP, CS, and edge rectification (WZP-CS-ER) [14];

— DWT based super resolution (DWT SR) [15];

- complex wavelet transform based super resolution (CWT SR) [5]. According to the quantitative and qualitative experimental results, the proposed technique over performs the aforementioned conventional and state-of-art techniques for image resolution enhancement.

\section{Proposed Image Resolution Enhancement}

In image resolution enhancement by using interpolation the main loss is on its high frequency components (i.e., edges), which is due to the smoothing caused by interpolation. In order to increase the quality of the super resolved image, preserving the edges is essential. In this work, DWT has been employed in order to preserve the high frequency components of the image. The redundancy and shift invariance of the DWT mean that DWT coefficients are inherently interpolable [9].

In this correspondence, one level DWT (with Daubechies 9/7 as wavelet function) is used to decompose an input image into different subband images. Three high frequency subbands (LH, HL, and HH) contain the high frequency components of the input image. In the proposed technique, bicubic interpolation with enlargement factor of 2 is applied to high frequency subband images. Downsampling in each of the DWT subbands causes information loss in the respective subbands. That is why SWT is employed to minimize this loss.

The interpolated high frequency subbands and the SWT high frequency subbands have the same size which means they can be added with each other. The new corrected high frequency subbands can be interpolated further for higher enlargement. Also it is known that in the wavelet domain, the low resolution image is obtained by lowpass filtering of the high resolution image [16]. In other words, low frequency subband is the low resolution of the original image. Therefore, instead of using low frequency subband, which contains less information than the original high resolution image, we are using the input image for the interpolation of low frequency subband image. Using input image instead of low frequency subband increases the quality of the super resolved image. Fig. 1 illustrates the block diagram of the proposed image resolution enhancement technique.

By interpolating input image by $\alpha / 2$, and high frequency subbands by 2 and $\alpha$ in the intermediate and final interpolation stages respectively, and then by applying IDWT, as illustrated in Fig. 1, the output image will contain sharper edges than the interpolated image obtained by interpolation of the input image directly. This is due to the fact that, the interpolation of isolated high frequency components in high frequency subbands and using the corrections obtained by adding high frequency subbands of SWT of the input image, will preserve more high frequency components after the interpolation than interpolating input image directly.

\section{RESUlTS AND Discussions}

Fig. 2 shows that super resolved image of Baboon's picture using proposed technique in (d) are much better than the low resolution 


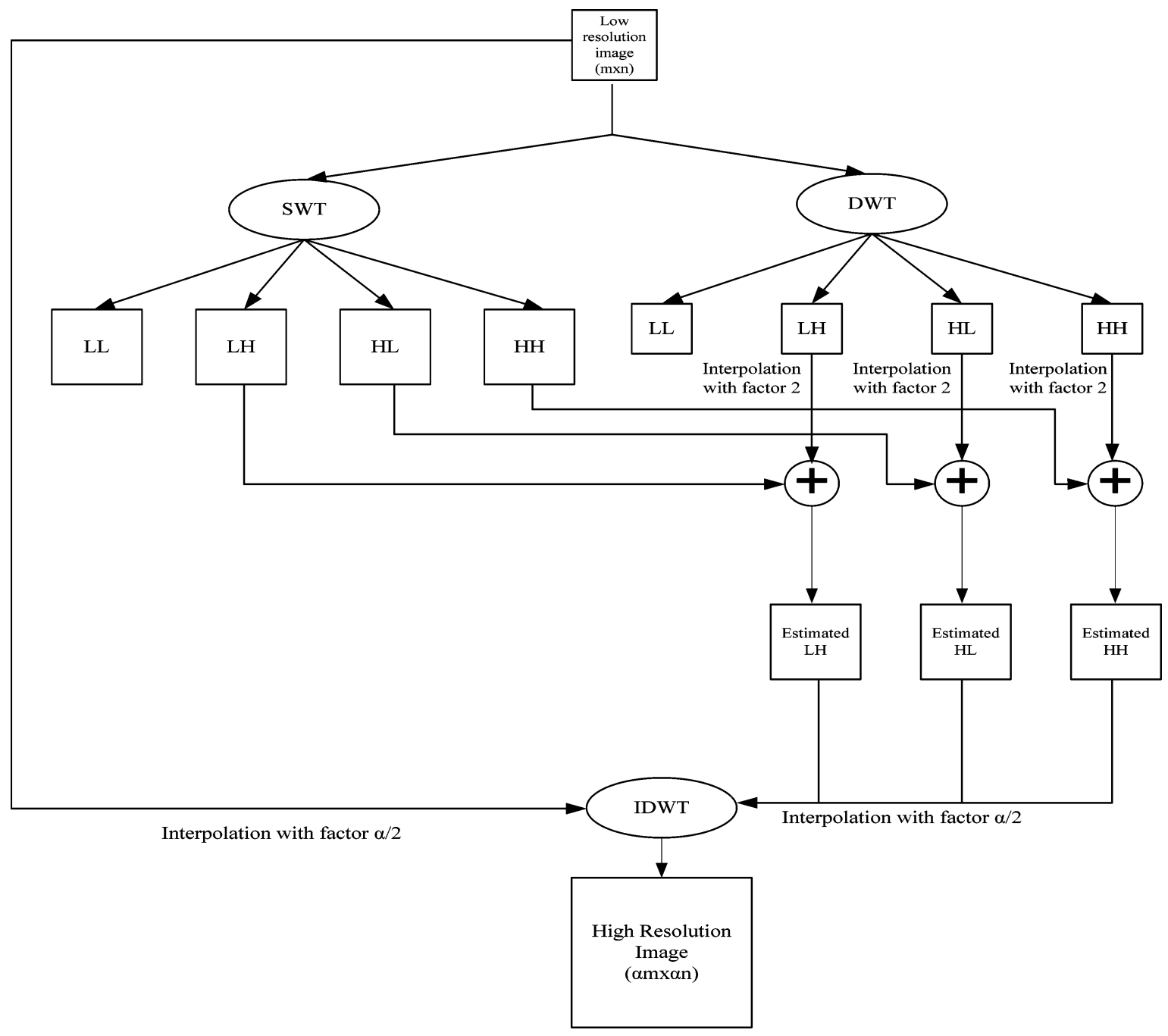

Fig. 1. Block diagram of the proposed super resolution algorithm.

image in (a), super resolved image by using the interpolation (b), and WZP (c). Note that the input low resolution images have been obtained by down-sampling the original high resolution images. In order to show the effectiveness of the proposed method over the conventional and state-of-art image resolution enhancement techniques, four well-known test images (Lena, Elaine, Baboon, and Peppers) with different features are used for comparison. Table I compares the PSNR performance of the proposed technique using bicubic interpolation with conventional and state-of-art resolution enhancement techniques: bilinear, bicubic, WZP, NEDI, HMM, HMM SR, WZP-CS, WZP-CS-ER, DWT SR, CWT SR, and regularity-preserving image interpolation. Additionally, in order to have more comprehensive comparison, the performance of the super resolved image by using SWT only (SWT-SR) is also included in the table. The results in Table I indicate that the proposed technique over-performs the aforementioned conventional and state-of-art image resolution enhancement techniques. Table I also indicates that the proposed technique over-performs the aforementioned conventional and state-of-art image resolution enhancement techniques.

\section{CONCLUSION}

This work proposed an image resolution enhancement technique based on the interpolation of the high frequency subbands obtained by DWT, correcting the high frequency subband estimation by using

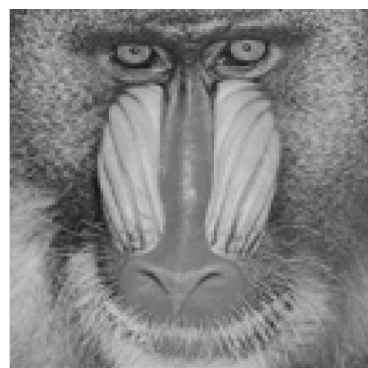

(a)

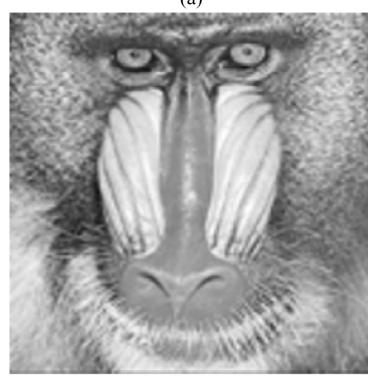

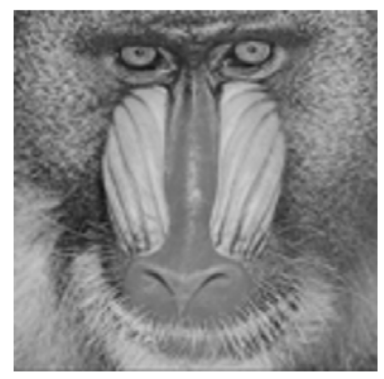

(b)

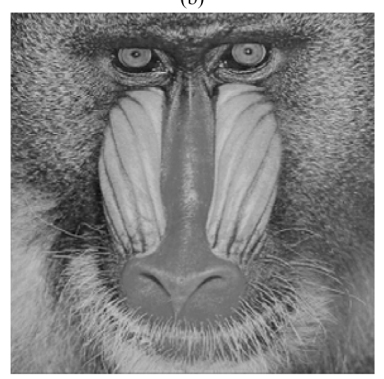

(d)
Fig. 2. (a) Original low resolution Baboon's image. (b) Bicubic interpolated image. (c) Super resolved image using WZP. (d) Proposed technique. 
TABLE I

PSNR (DB) RESULTS FOR RESOLUTION ENHANCEMENT FROM $128 \times 128$ TO $512 \times 512$ OF THE PROPOSED TECHNIQUE

COMPARED With THE CONVENTIONAL AND STATE-OF-ART IMAGE RESOLUTION ENHANCEMENT TECHNIQUES

\begin{tabular}{|c|c|c|c|c|}
\cline { 2 - 5 } \multicolumn{1}{c|}{} & \multicolumn{4}{c|}{ PSNR (dB) } \\
\hline Techniques $\backslash$ images & Lena & Elaine & Baboon & Peppers \\
\hline Bilinear & 26.34 & 25.38 & 20.51 & 25.16 \\
\hline Bicubic & 26.86 & 28.93 & 20.61 & 25.66 \\
\hline WZP (db. 9/7) & 28.84 & 30.44 & 21.47 & 29.57 \\
\hline $\begin{array}{c}\text { Regularity-Preserving } \\
\text { Image Interpolation[7] }\end{array}$ & 28.81 & 30.42 & 21.47 & 29.57 \\
\hline NEDI [10] & 28.81 & 29.97 & 21.18 & 28.52 \\
\hline HMM [11] & 28.86 & 30.46 & 21.47 & 29.58 \\
\hline HMM SR [12] & 28.88 & 30.51 & 21.49 & 29.60 \\
\hline WZP-CS [13] & 29.27 & 30.78 & 21.54 & 29.87 \\
\hline WZP-CS-ER [14] & 29.36 & 30.89 & 21.56 & 30.05 \\
\hline DWT SR [15] & 34.79 & 32.73 & 23.29 & 32.19 \\
\hline CWT SR [5] & 33.74 & 33.05 & 23.12 & 31.03 \\
\hline SWT SR & 32.01 & 31.25 & 22.74 & 29.46 \\
\hline Proposed Technique & $\mathbf{3 4 . 8 2}$ & $\mathbf{3 5 . 0 1}$ & $\mathbf{2 3 . 8 7}$ & $\mathbf{3 3 . 0 6}$ \\
\hline
\end{tabular}

SWT high frequency subbands, and the input image. The proposed technique uses DWT to decompose an image into different subbands, and then the high frequency subband images have been interpolated. The interpolated high frequency subband coefficients have been corrected by using the high frequency subbands achieved by SWT of the input image. An original image is interpolated with half of the interpolation factor used for interpolation the high frequency subbands. Afterwards all these images have been combined using IDWT to generate a super resolved imaged. The proposed technique has been tested on well-known benchmark images, where their PSNR and visual results show the superiority of proposed technique over the conventional and state-of-art image resolution enhancement techniques.

\section{ACKNOWLEDGMENT}

The authors would like to thank Dr. I. Selesnick from Polytechnic University for providing the DWT and SWT codes in MATLAB.

\section{REFERENCES}

[1] L. Yi-bo, X. Hong, and Z. Sen-yue, "The wrinkle generation method for facial reconstruction based on extraction of partition wrinkle line features and fractal interpolation," in Proc. 4th Int. Conf. Image Graph., Aug. 22-24, 2007, pp. 933-937.

[2] Y. Rener, J. Wei, and C. Ken, "Downsample-based multiple description coding and post-processing of decoding," in Proc. 27th Chinese Control Conf., Jul. 16-18, 2008, pp. 253-256.

[3] H. Demirel, G. Anbarjafari, and S. Izadpanahi, "Improved motionbased localized super resolution technique using discrete wavelet transform for low resolution video enhancement," in Proc. 17th Eur. Signal Process. Conf., Glasgow, Scotland, Aug. 2009, pp. 1097-1101.
[4] Y. Piao, I. Shin, and H. W. Park, "Image resolution enhancement using inter-subband correlation in wavelet domain," in Proc. Int. Conf. Image Process., 2007, vol. 1, pp. I-445-448.

[5] H. Demirel and G. Anbarjafari, "Satellite image resolution enhancement using complex wavelet transform," IEEE Geoscience and Remote Sensing Letter, vol. 7, no. 1, pp. 123-126, Jan. 2010.

[6] C. B. Atkins, C. A. Bouman, and J. P. Allebach, "Optimal image scaling using pixel classification," in Proc. Int. Conf. Image Process., Oct. 7-10, 2001, vol. 3, pp. 864-867.

[7] W. K. Carey, D. B. Chuang, and S. S. Hemami, "Regularity-preserving image interpolation," IEEE Trans. Image Process., vol. 8, no. 9, pp. 1295-1297, Sep. 1999.

[8] S. Mallat, A Wavelet Tour of Signal Processing, 2nd ed. New York: Academic, 1999.

[9] J. E. Fowler, "The redundant discrete wavelet transform and additive noise," Mississippi State ERC, Mississippi State University, Tech. Rep. MSSU-COE-ERC-04-04, Mar. 2004.

[10] X. Li and M. T. Orchard, "New edge-directed interpolation," IEEE Trans. Image Process., vol. 10, no. 10, pp. 1521-1527, Oct. 2001.

[11] K. Kinebuchi, D. D. Muresan, and R. G. Baraniuk, "Waveletbased statistical signal processing using hidden Markov models," in Proc. Int. Conf. Acoust., Speech, Signal Process., 2001, vol. 3, pp. 7-11.

[12] S. Zhao, H. Han, and S. Peng, "Wavelet domain HMT-based image super resolution," in Proc. IEEE Int. Conf. Image Process., Sep. 2003, vol. 2, pp. 933-936.

[13] A. Temizel and T. Vlachos, "Wavelet domain image resolution enhancement using cycle-spinning," Electron. Lett., vol. 41, no. 3, pp. 119-121, Feb. 3, 2005.

[14] A. Temizel and T. Vlachos, "Image resolution upscaling in the wavelet domain using directional cycle spinning," J. Electron. Imag., vol. 14, no. 4, 2005.

[15] G. Anbarjafari and H. Demirel, "Image super resolution based on interpolation of wavelet domain high frequency subbands and the spatial domain input image," ETRI J., vol. 32, no. 3, pp. 390-394, Jun. 2010.

[16] A. Temizel, "Image resolution enhancement using wavelet domain hidden Markov tree and coefficient sign estimation," in Proc. Int. Conf. Image Process., 2007, vol. 5, pp. V-381-384. 